EPJ Web of Conferences 41, 02036 (2013)

DOI: $10.1051 /$ epjconf/20134102036

(C) Owned by the authors, published by EDP Sciences, 2013

\title{
Time-Resolved Photoelectron Spectroscopy of Coupled Nuclear-Electronic Dynamics
}

\author{
M. Falge ${ }^{1}$, V. Engel ${ }^{1}$, and S. Gräfe ${ }^{2}$ \\ ${ }^{1}$ Universität Würzburg, Institut für Physikalische und Theoretische Chemie and Röntgen Research \\ Center for Complex Material Systems, Emil-Fischer Str. 42, 97074 Würzburg, Germany \\ ${ }^{2}$ Institute for Theoretical Physics, Vienna University of Technology, Wiedner Hauptstr. 8-10, A- \\ 1040 Vienna, Austria, email: Stefanie.Graefe@tuwien.ac.at
}

\begin{abstract}
We study the effect of nuclear-electron coupling on time-resolved photoelectron spectra, employing a model system which allows to directly comparing spectra resulting from the adiabatic approximation with those obtained within a non-BornOppenheimer description.
\end{abstract}

\section{Introduction}

Using femtosecond time-resolved photoelectron spectroscopy (TR-PES), nuclear dynamics in molecules can be monitored [1]. The photoelectron signal can be either resolved in energy [2,3] and/or in the angular distribution (for a review see, e.g., [4,5]) and has been studied both experimentally and theoretically. Calculations of TR-PES are typically based on the approach first developed by Seel and Domcke [6], and later by Meier and Engel [7], and do not include the electron dynamics explicitly. McKoy et al. have derived a mixed quantum chemical - quantum dynamical approach to treat (nonadiabatic) electronic dynamics [8]. Here, we suggest an alternative approach to treat nuclear-electron coupling in a system of reduced dimensionality [9] which allows describing nonadiabatic dynamics. The system consists of two ions, fixed at distances $\pm L / 2$, an additional ion and an electron moving in one dimension between the fixed ions. The model system allows for a description of nonadiabatic wavepacket dynamics [10]. Its Hamiltonian is given by:

$$
\begin{aligned}
& H_{0}=\frac{p^{2}}{2}+\frac{P^{2}}{2 M}+V(r, R), \quad \text { with } \\
& V(r, R)=\frac{1}{|L / 2-R|}+\frac{1}{|L / 2+R|}-\frac{\operatorname{erf}\left(\left|L / 2-r / R_{f}\right|\right)}{|L / 2-r|}-\frac{\operatorname{erf}\left(\left|L / 2+r / R_{f}\right|\right)}{|L / 2+r|} \frac{\operatorname{erf}\left(\left|R-r / R_{c}\right|\right)}{|R-r|},
\end{aligned}
$$

where $M$ is the nuclear mass and $(r, R),(p, P)$ denote the electronic and nuclear coordinate and momentum operators, respectively. The origin of the coordinate system is at the midpoint between the two fixed ions. In the calculation, we chose $L=10 \AA$, and $R_{f}=1.5 \AA$. The parameter $R_{c}$ determines the electron-nuclear interaction and allows to realize different coupling cases, ranging from weak (almost adiabatic motion) to strong coupling (diabatic motion). We numerically solve the coupled nuclear electronic Schrödinger equation (2D), as well as the nuclear Schrödinger equation in a fixed electronic state $|n\rangle$ within the Born-Oppenheimer approximation (BO) on a grid using the splitoperator method. The XUV interaction for the BO calculations is treated within first order perturbation theory, for details see [11].

This is an Open Access article distributed under the terms of the Creative Commons Attribution License 2.0, which permits unrestricted use, distribution, and reproduction in any medium, provided the original work is properly cited. 


\section{EPJ Web of Conferences}

\section{Results and Discussion}

Initially, a nuclear wavepacket is launched on an excited electronic state. A XUV pulse with a wavelength of $60 \mathrm{~nm}(20.7 \mathrm{eV})$ and $4 \mathrm{fs}$ Gaussian full width at half maximum ionizes the system at a delay time $T$. Fig. 1 displays the photoelectron spectra $\sigma\left(E_{p}, T\right)$ of the $2 \mathrm{D}$ calculation and the BO calculation $\sigma^{B O}\left(E_{p}, T\right)$ for both, the weak and strong coupling cases. Within the adiabatic picture, the energy $\varepsilon_{n}^{\max }(T)$ where the spectrum, resulting from ionization of state $|n\rangle$ exhibits a maximum, can be estimated: For an initial wave packet localized around its center $\langle R\rangle_{T}$ at time $T$, one finds [12]:

$$
\varepsilon_{n}^{\max }\left(\langle R\rangle_{T}\right)=\omega_{X U V}-\left[V_{d}\left(\langle R\rangle_{T}\right)-V_{n}\left(\langle R\rangle_{T}\right)\right] .
$$

We find that for the weak coupling case (left panels), the adiabatic treatment gives results which agree well with the full numerical solutions. There is, however, a difference between the two spectra: the spectra originating from the $2 \mathrm{D}$ calculation feature an intensity variation as a function of delay time $T$, which is not found in the $\mathrm{BO}$ calculation. This difference can be explained by the dependence of the transition dipole moment on both, the nuclear coordinate and the photoelectron energy. We verified this in a calculation which includes a transition dipole moment, yielding a spectrum $\sigma^{B O}\left(E_{p}, T\right)$ which shows a similar intensity variation as is seen in $\sigma\left(E_{p}, T\right)$ (not shown). Also the total photoelectron yield (the integral over the photoelectron spectrum), does depend on the delay time $T$. This is not the case in the adiabatic spectrum employing a constant dipole moment $[6,13]$. This difference is therefore the result of a non-Condon effect for the transition dipole moment.
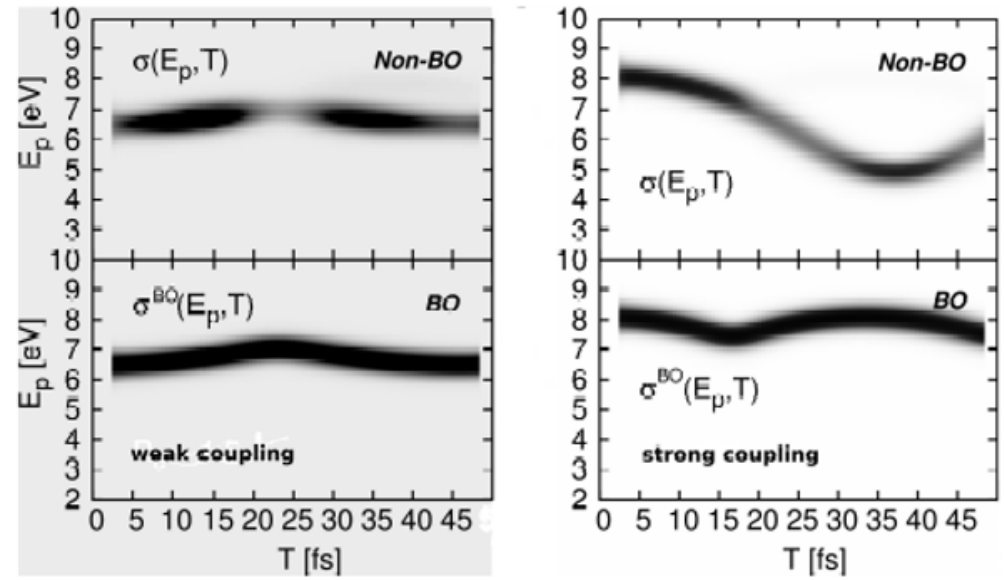

Figure 1 Time-resolved photoelectron spectra from the non-Born-Oppenheimer (2D) coupled nuclear-electronic dynamics $\sigma\left(E_{p}, T\right)$ (upper panels) and from the adiabatic approximations $\sigma^{B O}\left(E_{p}, T\right)$ (lower panels). Left panels: weak coupling $\left(R_{c}=1.5 \AA\right)$, right panels: strong coupling $\left(R_{c}=2.5 \AA\right)$, as labeled (adapted from Ref. [11]).

In the case of strong coupling, the adiabatic approximation breaks down and the photoelectron spectra originating from the full numerical calculation and the BO calculation differ substantially (Fig. 1, right column). This can be explained by an almost diabatic wavepacket motion: at times larger than $15 \mathrm{fs}$, most of the population is transferred to a lower electronic state with higher ionization potential, which leads to a shift of the intensity of the photoelectron spectrum to lower energies. The nonadiabatic coupling strength depends strongly on the nuclear mass $M$, as can be seen regarding the nonadiabatic coupling elements between the electronic states $|n\rangle$ and $|m\rangle$

$$
T_{m n}^{(j)}(R)=\frac{1}{M} \int d r \varphi_{m}(r, R) \frac{\partial^{j}}{\partial R^{j}} \varphi_{n}(r, R) .
$$

With increasing mass $M$, the efficiency for nonadiabatic transitions decreases. We have calculated photoelectron spectra for the strong coupling case varying the nuclear mass $M$, see Fig. 2 . 


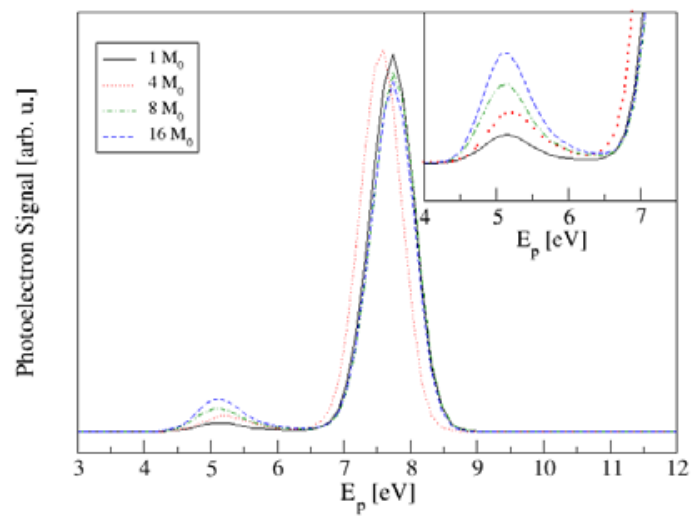

Figure 2 Photoelectron spectra from the non-Born-Oppenheimer (2D) case $\sigma\left(E_{p}, T\right)$ calculated for multiples of the hydrogen mass $\left(\mathrm{M}_{0} \approx 2000\right.$ a.u.) for delay times $T$ where the non-adiabatic transition takes place (strong coupling case). The inset shows the enlarged peak at lower energies (adapted from Ref. [11]).

In Fig. 2, two peaks can be identified, where one peak at lower energies originates from ionization from the lower electronic state, and the peak at higher energies from ionization from the upper state, being located at similar energies as for the BO case. The relative peak height directly mirrors the strength of the non-adiabatic transition. Thus, the photoelectron spectra reflect the nonadiabatic dynamics, providing an estimate of the nonadiabatic transition amplitudes.

\section{Conclusions}

We have examined the effect of coupled nuclear-electronic dynamics on time-resolved photoelectron spectra in a simple model system by comparing spectra calculated from a full numerical description of the coupled dynamics with an approximate treatment within the BO approximation. We have found that for weak coupling, the approximation to derive spectra using the BO approximation and time-dependent perturbation theory works well, although even for this case, differences in the spectra can be seen. These differences are mainly due to the neglect of the dependence of the transition dipole moment on the nuclear coordinate. For the strong coupling case, the adiabatic approximation breaks down and the spectra differ substantially. In the photoelectron distributions, two peaks can be detected originating from contributions of different electronic states. The relative heights of these peaks provide an estimate of the nonadiabatic transition amplitudes, see Ref. [11].

Acknowledgements: M. F. acknowledges financial support by the state of Bavaria (Bayersisches Eliteförderungsgesetz). S. G. acknowledges financial support by the Austrian Science Fund FWF, project number V193-N16.

1. A. H. Zewail, Femtochemistry, Vols. I, II (World Scientific, Singapore, 1994).

2. A. Stolow, A. E. Bragg, and D. M. Neumark, Chem. Rev. 104, 1719 (2004).

3. M. Wollenhaupt, V. Engel, and T. Baumert, Annu. Rev. Phys. Chem. 56, 25 (2005).

4. K. L. Reid, Annu. Rev. Phys. Chem. 54, 397 (2003).

5. T. Seideman, Rev. Phys. Chem. 53, 41 (2002).

6. M. Seel and W. Domcke, J. Chem. Phys. 95, 7806 (1991).

7. C. Meier and V. Engel, Chem. Phys. Lett. 212, 691 (1993).

8. Y. Arasaki, K. Takatsuka, K. Wang, and V. McKoy, Chem. Phys. Lett. 302, 363 (1999).

9. S. Shin and H. Metiu, J. Chem. Phys. 102, 9285 (1995); ibid., J. Phys. Chem. 100, 7867 (1996).

10. M. Erdmann and V. Engel, J. Chem. Phys. 120, 158 (2004).

11. M. Falge, V. Engel, S. Gräfe, J. Chem. Phys. 134, 184307 (2011).

12. M. Braun, C. Meier, V. Engel, J. Chem. Phys. 103, 7907 (1995).

13. V. Engel, Chem. Phys. Lett. 178, 130 (1991). 